\title{
Magnetic Order and Other Phase Transitions in Mixed-Valent YbPd
}

\author{
R. Pott, ${ }^{(a)}$ W. Boksch, G. Leson, B. Politt, H. Schmidt, A. Freimuth, K. Keulerz, J. Langen, \\ G. Neumann, F. Oster, J. Röhler, U. Walter, P. Weidner, and D. Wohlleben \\ II. Physikalisches Institut, Universität Köln, D-5000 Köln 41, Federal Republic of Germany \\ (Received 22 August 1984)
}

\begin{abstract}
Specific heat, thermal expansion, and electrical resistivity show that $\mathrm{YbPd}$ undergoes four phase transitions at $0.5,1.9,105$, and $125 \mathrm{~K}$. From magnetic-susceptibility measurements the transition at $0.5 \mathrm{~K}$ is found to be magnetic. The quasielastic magnetic linewidth is large as usual for mixed valence, but decreases with temperature and is smaller than $k_{\mathrm{B}} T$ everywhere. The magnetic order of YbPd occurs at a fractional $\mathrm{Yb}$ valence of $v=2.8$ (measured by $L_{\mathrm{III}}$ absorption), i.e., near configurational crossover of $4 f^{13}$ and $4 f^{14}$.
\end{abstract}

PACS numbers: $75.30 .-\mathrm{m}$

Magnetic order of $\mathrm{Yb}$ in metals is rare. To our knowledge the only certain case was $\mathrm{YbBe}_{13} 1,2$ $\left(T_{\text {Neel }}=1.28 \mathrm{~K}, \mu_{\text {eff }}=4.54 \mu_{\mathrm{B}}\right)$ until recently. This rarity of magnetic order may be blamed on the tendency of $\mathrm{Yb}$ to go into a mixed-valent state. However, on the other end of the lanthanide series Ce has the same tendency and yet shows magnetic order quite frequently. Since compounds with $\mathrm{Ce}$ have been studied much more frequently than compounds with $\mathrm{Yb}$, the rarity of magnetic order in $\mathrm{Yb}$ compounds may simply be a matter of insufficient statistics. Indeed, three new cases of magnetically ordering $\mathrm{Yb}$ compounds have recently been found: $\mathrm{YbPd}, \mathrm{Yb}_{3} \mathrm{Pd}_{4},{ }^{3}$ and $\mathrm{YbIr}_{2}{ }^{4}$

In this paper we report a thorough study of $\mathrm{YbPd}$. This compound turns out to be a very interesting system, because the magnetic order occurs clearly at fractional valence, near configurational crossover of $4 f^{13}$ and $4 f^{14}$.

The major problem in the initial stages of this work was the preparation of single phase $\mathrm{YbPd}$. The ingots were heated in a welded $\mathrm{Ta}$ crucible by induction. Only minor deficiency of $\mathrm{Yb}$ leads to the formation of

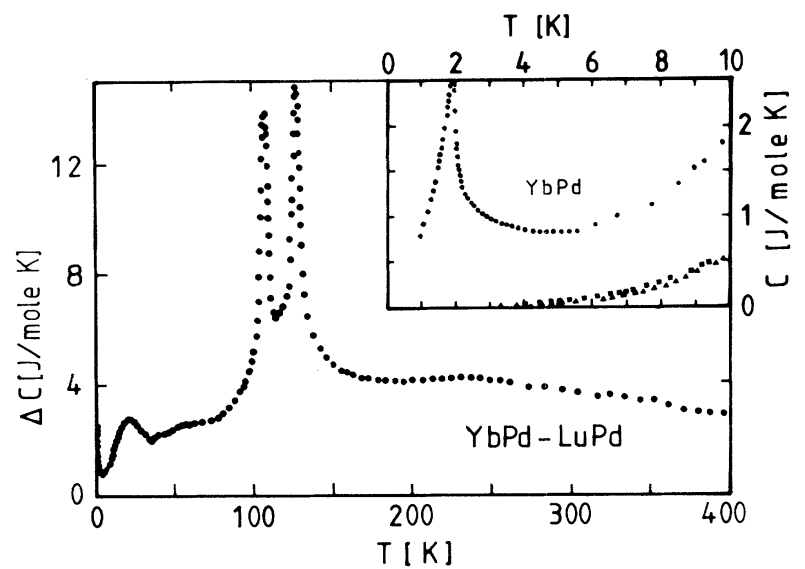

FIG. 1. Difference $\Delta C$ of the specific heats of $Y b P d$ and LuPd vs temperature. Inset: Specific heats of YbPd (circles), LuPd (squares), and CaPd (triangles) at low temperatures.
$\mathrm{Yb}_{3} \mathrm{Pd}_{4}$, which we also found to order magnetically. ${ }^{3}$ According to our experience a 3\% surplus of $\mathrm{Yb}$ is the proper procedure to obtain stoichiometric YbPd. All samples (including the isostructural reference compounds LuPd and CaPd) were characterized by Debye-Scherrer patterns and microsections and showed the cubic $\mathrm{CsCl}$ structure. ${ }^{5}$

We have measured specific heat, thermal expansion, thermopower, and neutron inelastic magnetic scattering between $T \simeq 1.5$ and $350 \mathrm{~K}$ and electrical resistivity and magnetic susceptibility between $40 \mathrm{mK}$ and 300 $\mathrm{K}$ (Figs. 1-5).

The lattice-constant anomaly of $\mathrm{YbPd}$ observed between 77 and $1000 \mathrm{~K}$ (as compared with CaPd and LuPd) was used by Iandelli and Palenzona ${ }^{5}$ to classify YbPd as clearly mixed valent.

Our low-temperature specific heat of YbPd is much larger than that of the reference compounds LuPd and $\mathrm{CaPd}$ (inset of Fig. 1). The difference $\Delta C$ of the specific heats of YbPd and LuPd shows four anomalies at $1.9, \simeq 17,105$, and $125 \mathrm{~K}$ (Fig. 1), which all can be found again in the difference of the thermal expansion coefficients $\Delta \alpha$ (Fig. 2) and in the resistivity $\rho$ (Fig. 3).

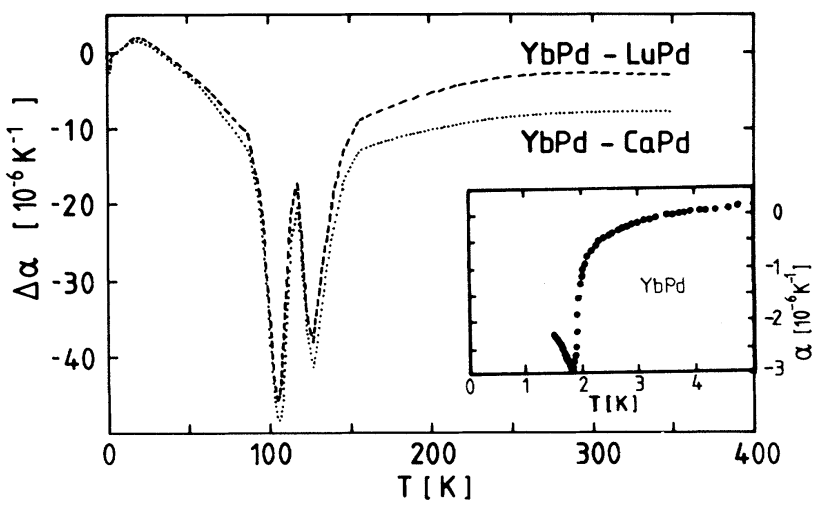

FIG. 2. Difference $\Delta \alpha$ of the thermal expansion coefficients of $\mathrm{YbPd}$ and $\mathrm{LuPd} / \mathrm{CaPd}$ vs temperature. Inset: $\alpha$ at low temperatures. 


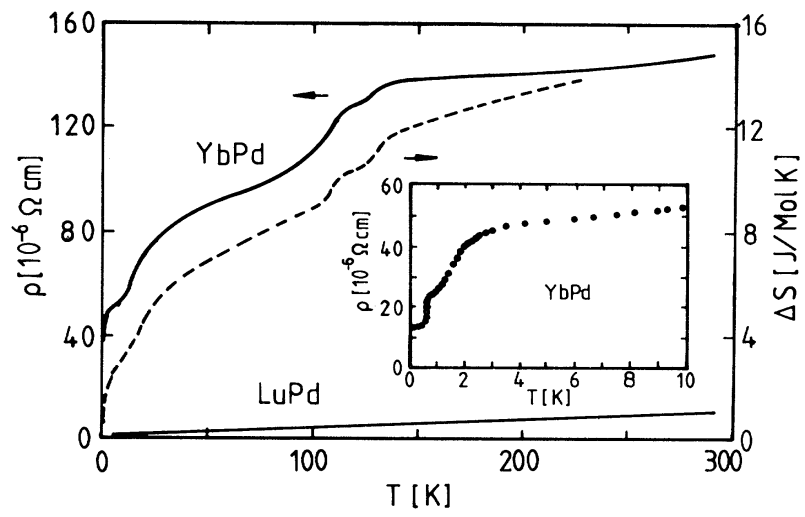

FIG. 3. Resistivity $\rho$ of YbPd and LuPd vs temperature and difference of entropies $\Delta S$ of YbPD and LuPD vs temperature. Inset: $\rho$ at low temperatures.

$\Delta \alpha$ is negative between 1.5 and $400 \mathrm{~K}$. This is typical for an intermediate-valence (IV) $\mathrm{Yb}$ compound, where with increasing temperature the valence is usually shifting towards the trivalent state $\left(4 f^{13}\right)$ with the smaller volume. The unusual sharp spikes of $\Delta \alpha$ at 105 and $125 \mathrm{~K}$ could indicate a structural phase transition, but careful temperature-dependent $\mathrm{x}$-ray measurements did not give any hint in this direction. However, the lines are all quite broad.

With a room-temperature valence of 2.83 or 2.79 [calculated from lattice parameters with Vegard's law (curve $b$ ) and with the "sagging curve" model (curve c) ${ }^{6}$ ] we have tried to extract the valence and its temperature dependence from the lattice constant and the thermal expansion anomaly (Fig. 6). Below about 110 $\mathrm{K}$ the values differ substantially from those obtained from $L_{\text {III }}$ absorption edges (curve $a$ ) (Fig. 6). We interpret this discrepancy as another indication for structural phase transitions at 105 and $125 \mathrm{~K}$, which seem to cause a considerable volume change without valence change, thus falsifying the valence determina-

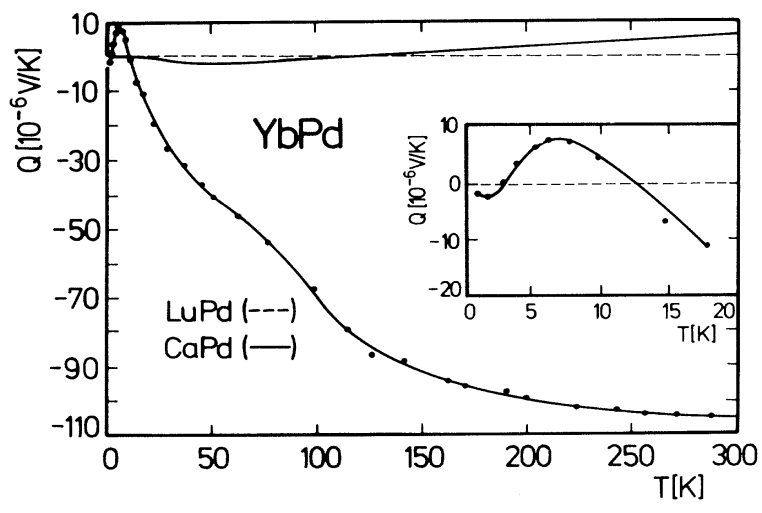

FIG. 4. Thermopowers $Q$ vs temperature for YbPd, LuPd, and CaPd. Inset: $Q$ at low temperatures.

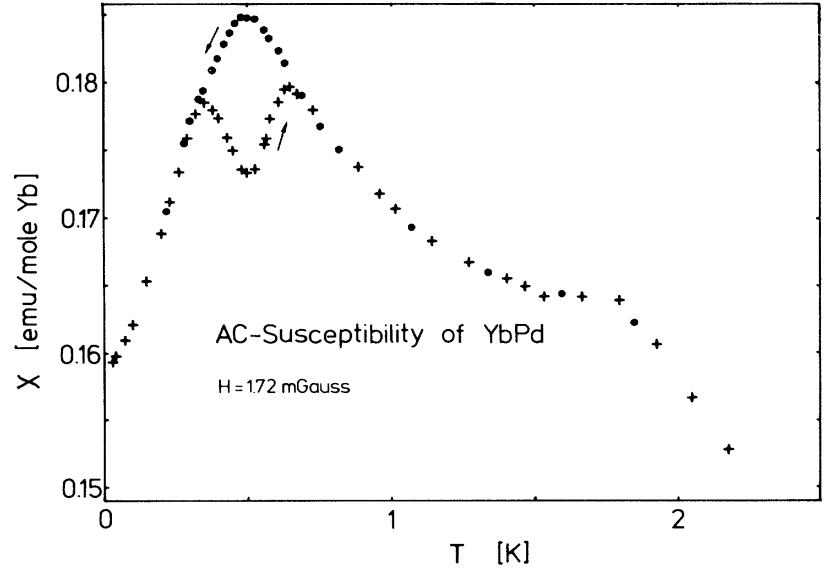

FIG. 5. Susceptibility $\chi$ vs temperature for YbPd at low temperatures (arrows indicate cooling and heating curves).

tion via the volume anomaly by $\delta v_{s}$ of order $10 \%$ (Fig. 6). Another falsification of the valence determination via the volume anomaly is due to the strong temperature dependence of the fluctuation temperature (see below), which causes the finite slope of $v(T)$ above about $130 \mathrm{~K} .^{7}$ Both valence measurements show that YbPd is clearly mixed valent, which is confirmed by all other measurements carried through at our institute (magnetic susceptibility, neutron inelastic scattering, magnetostriction, thermal conductivity, and pointcontact spectroscopy).

The thermopower of YbPd is uncommonly large and negative over a wide temperature range (Fig. 4). Note the unusual behavior at low temperatures, i.e., the two sign reversals at 3 and $12 \mathrm{~K}$ (inset in Fig. 4). The negative thermopower and also the resistivity are quite similar to the entropy difference $\Delta S$. In the latter case the similarity is particularly striking over the entire temperature range of the measurement.

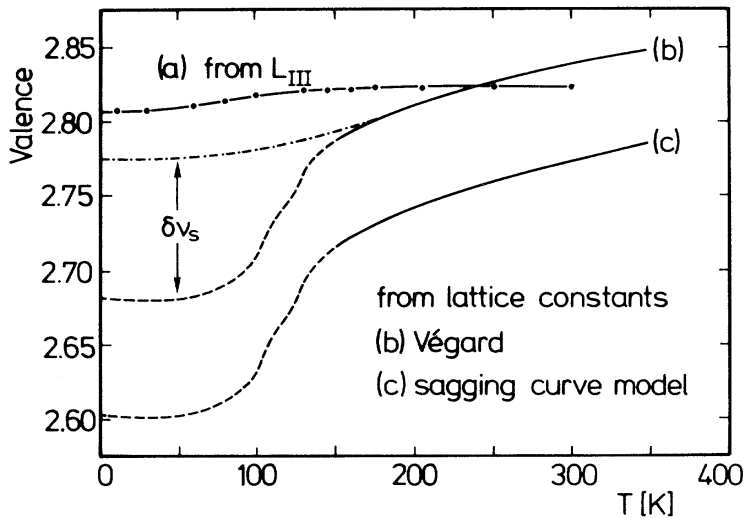

FIG. 6. Valence $v$ vs temperature as derived from $L_{\text {III }}$ absorption (curve $a$ ), Vegard's rule (curve $b$ ), and "sagging curve" model (curve $c$ ) (see text). 
The low-temperature resistivity shows two distinct anomalies at 1.9 and $0.5 \mathrm{~K}$ (inset in Fig. 3). The sharp decrease (together with the behavior of specific heat and thermal expansion at $1.9 \mathrm{~K}$ ) indicates that both anomalies are caused by electronic phase transition.

In order to characterize these low-temperature phase transitions better we measured the magnetic susceptibility (inductively) down to $40 \mathrm{mK}$ in a weak magnetic field of $H=1.72 \mathrm{mG}$ (Fig. 5). The distinct anomaly of $\chi$ at $0.5 \mathrm{~K}$ shows that we have magnetic ordering at this temperature (probably antiferromagnetic). This result is confirmed by a rough estimate of the ordering temperature, which can be obtained by de Gennes scaling from the ordering temperature of $\mathrm{Gd}^{3+}$ in $\operatorname{GdPd}\left(T_{\mathrm{N}}=38.6 \mathrm{~K}\right)$. This yields $T_{\mathrm{N}}=0.8 \mathrm{~K}$ for $100 \%$ $\mathrm{Yb}^{3+}$ and $T_{\mathrm{N}}=0.64 \mathrm{~K}$ for $80 \% \mathrm{Yb}^{3+}$. The large specific heat near the phase transition makes it difficult to measure the actual temperature of the sample near the ordering point during the cooling and heating cycles in our cryostat. This may be the reason for the unusual hysteresis loop in the magnetic susceptibility between 0.3 and $0.7 \mathrm{~K}$.

At this point one should ask: How can magnetic ordering appear in such a strongly mixed-valent compound? (At configurational crossover, i.e., at energetic degeneracy of $\mathrm{Yb}^{2+}$ and $\mathrm{Yb}^{3+}$, the valence is 2.89). This question was answered in part by measurements of the quasielastic linewidth of the neutron spectra down to $1.4 \mathrm{~K}$ that show that the fluctuation temperature $T_{f}$ is everywhere smaller than $k_{\mathrm{B}} T$ (Fig. 7). (At $300 \mathrm{~K}$ the quasielastic linewidth is between 5 and 15 meV.) At low temperatures there also appear inelastic transitions between 5 and $15 \mathrm{meV}$ as one would expect, for instance, when the quasielastic linewidth becomes smaller than the usual crystal-field splittings of $\mathrm{Yb}^{3+}{ }^{8} \quad T_{f}<T$ implies that at the ordering temperature predicted by de Gennes scaling the magnetic ordering energy $k_{\mathrm{B}} T_{\mathrm{N}}$ is larger than the valence fluctuation energy $k_{\mathrm{B}} T_{f}$. The more pertinent question then is: Why does $T_{f}$ drop so dramatically with temperature in $\mathrm{YbPd}$, while in other mixed-valence compounds with nearly the same fractional valence $T_{f}$ is fairly temperature independent and large compared to $T_{\mathrm{N}}$ extrapolated from de Gennes scaling? $\left(T_{f} \simeq 40 \mathrm{~K}\right.$ in $\mathrm{YbCu}_{2} \mathrm{Si}_{2}{ }^{9} ; T_{f} \simeq 50 \mathrm{~K}$ in $\mathrm{YbCuAl}^{10}$ ).

The anomaly of $\chi$ at $1.9 \mathrm{~K}$ is not as pronounced as the one at $0.5 \mathrm{~K}$. One may doubt whether the phase transition at this temperature is due to magnetic order. Rather we might observe quadrupolar ordering, as was reported for $\mathrm{TmZn}^{11}$ and $\mathrm{UPd}_{3}{ }^{12}$

$\mathrm{YbPd}$ is a mixed-valence compound with a valence changing from $v=2.82_{2}$ at $300 \mathrm{~K}$ to $v=2.80_{5}$ near $T=0$. We have detected four phase transitions, two of which, at 105 and $125 \mathrm{~K}$, should be structural, although this could not be confirmed, while two others at 1.9 and $0.5 \mathrm{~K}$ are electronic. The transition at $1.9 \mathrm{~K}$

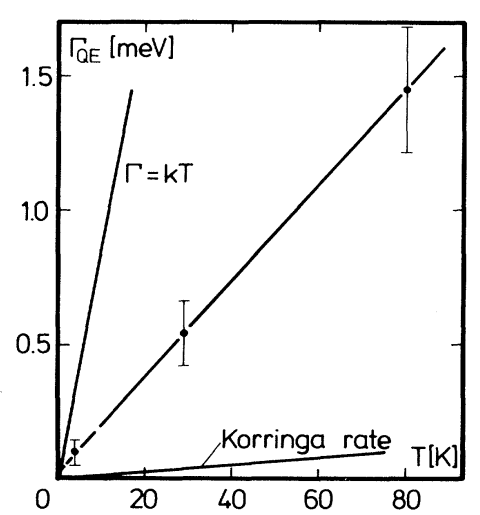

FIG. 7. Temperature dependence of the quasielastic magnetic linewidth $\Gamma$ (half width at half maximum) of $\mathrm{YbPd}$ below $80 \mathrm{~K} . \Gamma$ is much larger than that due to the usual electronic Korringa exchange mechanism but remains smaller than $k_{\mathrm{B}} T$.

may be due to quadrupolar ordering; the one at $0.5 \mathrm{~K}$ is due to magnetic order.

While all these phase transitions are very unusual, the magnetic one is of the greatest interest in principle, because it occurs in a clearly mixed-valent state. Up to now the absence of magnetic order in mixed valence compounds with $\mathrm{Ce}, \mathrm{Eu}, \mathrm{Sm}$, and $\mathrm{Yb}$ and its existence in strongly mixed valent $\mathrm{TmSe}^{13}$ has been rationalized ${ }^{14}$ by the observation that $\mathrm{Tm}$ has magnetic moments in the Hund's-rule ground states of both adjacent valence states ( $4 f^{12}$ and $4 f^{13}$ ), while in all the other cases one of the Hund's-rule ground states is a nonmagnetic singlet. One might argue that this rule has been violated already several times in the past [e.g., in $\mathrm{CeAl}_{2}$ and $\mathrm{Eu}\left(\mathrm{Pd}_{1-x} \mathrm{Au}_{x}\right) \mathrm{Si}_{2}{ }^{15}$ ], were it not for the fact that the magnetic order of these compounds is not usually associated with mixed valence, because the deviation from integral valence seems to be very small in these compounds. Thus the magnetic order of $\mathrm{YbPd}$ is of very special importance in this context. It confirms a different rule: Whenever the fluctuation temperature, for whatever reason, is smaller than the magnetic ordering temperature expected on the basis of de Gennes scaling from stable valent reference compounds and of the dilution effect implied in fractional valence, magnetic ordering occurs and vice versa.

We thank W. Scheffler for his assistance in the susceptibility measurements. This work was supported by the Deutsche Forschungsgemeinschaft through SFB 125 .

(a) Present address: Bayer AG, ZF-TPEG, Rheinuferstrasse, D-4150 Krefeld 11, Federal Republic of Germany. 
${ }^{1}$ G. Heinrich, J. P. Kappler, and A. Meyer, Phys. Lett. 74A, 121 (1979).

${ }^{2}$ G. V. Eynatten, C. F. Wang, N. S. Dixon, L. S. Fritz, and S. S. Hanna, Z. Phys. B 51, 37 (1983).

${ }^{3}$ B. Politt, D. Dürkop, and P. Weidner, in Proceedings of the International Conference on Valence Fluctuations, August 1984 (to be published).

${ }^{4}$ J. O. Willis, J. L. Smith, and Z. Fisk, in Ref. 3.

${ }^{5}$ A. Iandelli and A. Palenzona, Rev. Chim. Min. 10, 303 (1973).

${ }^{6}$ G. Neumann, R. Pott, J. Röhler, W. Schlabitz, D. Wohlleben, and H. Zahel, in Valence Instabilities, edited by P. Wachter and H. Boppart (North-Holland, Amsterdam, 1982), p. 87.

${ }^{7} \mathrm{G}$. Neumann et al., to be published.

${ }^{8} \mathrm{U}$. Walter, private communication.

${ }^{9}$ E. Holland-Mortiz, D. Wohlleben, and M. Löwenhaupt, Phys. Rev. B 25, 7482 (1973).
${ }^{10}$ W. C. M. Mattens, PhD. thesis, University of Amsterdam, 1980 (unpublished).

${ }^{11}$ P. M. Levy, P. Morin, and D. Schmitt, Phys. Rev. Lett. 42, 1417 (1979).

${ }^{12}$ D. Shaltiel, Phys. Rev. A 135, 1346 (1964). A. F. Marray and W. L. Buyers, in Crystal Electric Fields and Structural Effects in $f$-Electron Systems, edited by J. E. Crow, R. P. Guertin, and T. W. Mihalicin (Plenum, New York, 1980), p. 257; P. Zablinski et al., op. cit., p. 259.

${ }^{13}$ E. Bucher, A. C. Gossard, J. P. Maita, and A. S. Cooper in Proceedings of the Eighth Conference on Rare Earth Research, edited by T. A. Henry and R. E. Henry (U.S. GPO, Washington D.C., 1970), p. 74.

${ }^{14}$ C. M. Varma, in Proceedings of the U.S.-Japan Seminar on Physical Properties of Rare Earth Magnetic Semiconductors, Sendai, Japan, 1977 (unpublished).

${ }^{15}$ C. U. Segre, M. Croft, J. A. Hodges, V. Margai, L. C. Gupta, and R. D. Parks, Phys. Rev. Lett. 49, 1063 (1979). 